\title{
Homologs of the Hh signalling network in C. elegans*
}

Thomas R. Bürglin, Department of Biosciences and Nutrition and Center for Genomics and Bioinformatics, Karolinska Institutet and Life Sciences, Södertörns University College, Huddinge, Sweden

Patricia E. Kuwabara ${ }^{\S}$, Department of Biochemistry, University of Bristol, Bristol, UK BS8 1TD

\section{Table of Contents}

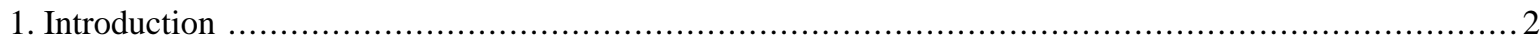

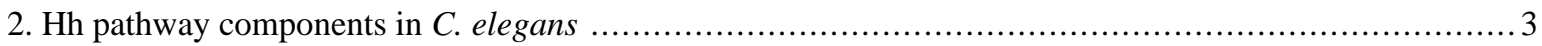

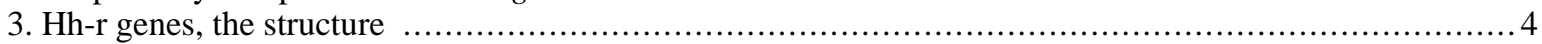

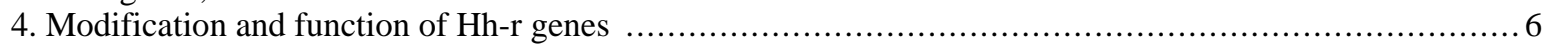

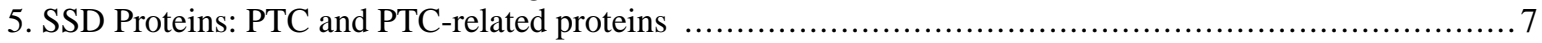

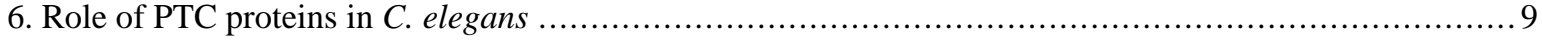

7. Other SSD proteins provide clues to PTC and PTR function ..............................................

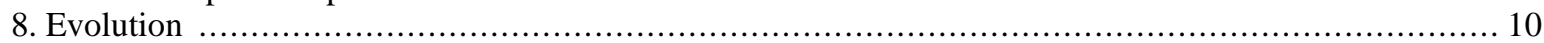

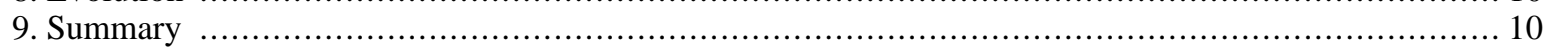

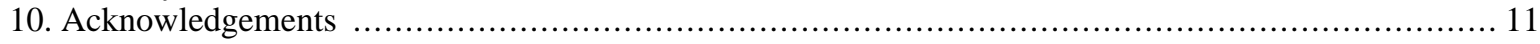

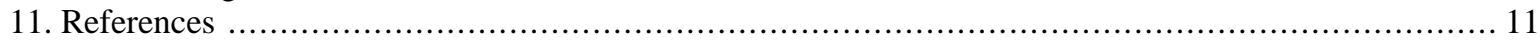

Abstract

In Drosophila and vertebrates, Hedgehog (Hh) signalling is mediated by a cascade of genes, which play essential roles in cell proliferation and survival, and in patterning of the embryo, limb buds and organs. In $C$. elegans, this pathway has undergone considerable evolutionary divergence; genes encoding homologues of key pathway members, including Hh, Smoothened, Cos2, Fused and Suppressor of Fused, are absent. Surprisingly, over sixty proteins (i.e. WRT, GRD, GRL, and QUA), encoded by a set of genes collectively referred to as the Hh-related genes, and two co-orthologs (PTC-1,-3) of fly Patched, a Hh receptor, are present in $C$. elegans. Several of the Hh-related proteins are bipartite and all can potentially generate peptides with signalling activity, although none of these peptides shares obvious sequence similarity with Hh.

*Edited by Iva Greenwald. Last revised February 16, 2005. Published January 28, 2006. This chapter should be cited as: Bürglin, T. R. and Kuwabara, P. E. Homologs of the Hh signalling network in C. elegans (January 28, 2006), WormBook, ed. The C. elegans Research Community, WormBook, doi/10.1895/wormbook.1.76.1, http://www.wormbook.org.

Copyright: () 2006 Thomas R. Bürglin and Patricia E. Kuwabara. This is an open-access article distributed under the terms of the Creative Commons Attribution License, which permits unrestricted use, distribution, and reproduction in any medium, provided the original author and source are credited.

${ }^{\S}$ To whom correspondence should be addressed. E-mail: p.kuwabara@bristol.ac.uk 
In addition, the ptc-related (ptr) genes, which are present in a single copy in Drosophila and vertebrates and encode proteins closely related to Patched, have undergone an expansion in number in nematodes. A number of functions, including roles in molting, have been attributed to the $C$. elegans Hh-related, PTC and PTR proteins; most of these functions involve processes that are associated with the trafficking of proteins, sterols or sterol-modified proteins. Genes encoding other components of the Hh signalling pathway are also found in C. elegans, but their functions remain to be elucidated.

\section{Introduction}

The Drosophila hedgehog (hh) mutant was identified by Nüsslein-Volhard and Wieschaus in their classic genetic screen that led to the identification of key embryonic segment polarity genes (Nüsslein-Volhard and Wieschaus, 1980). Subsequently, the single Drosophila and the multiple vertebrate Hh proteins, Sonic Hedgehog (Shh), Indian Hedgehog (Ihh) and Desert Hedgehog (Dhh), have collectively proven to be key effectors in patterning the embryo, limb buds and neural tube, organ formation, cell proliferation, axon guidance and cell survival (for reviews, see Ingham and McMahon, 2001; Lum and Beachy, 2004). Mutations in genes constituting the Hh signalling pathway are also implicated in various human syndromes and cancers (Bale, 2002; Beachy et al., 2004; Pasca di Magliano and Hebrok, 2003; Taipale and Beachy, 2001). Details of the pathway are summarised in Figure 1 (Ingham and McMahon, 2001; Lum and Beachy, 2004). The various proteins involved in the Hh and Ptc signalling pathway, including those present in C. elegans, are listed in Table 1.

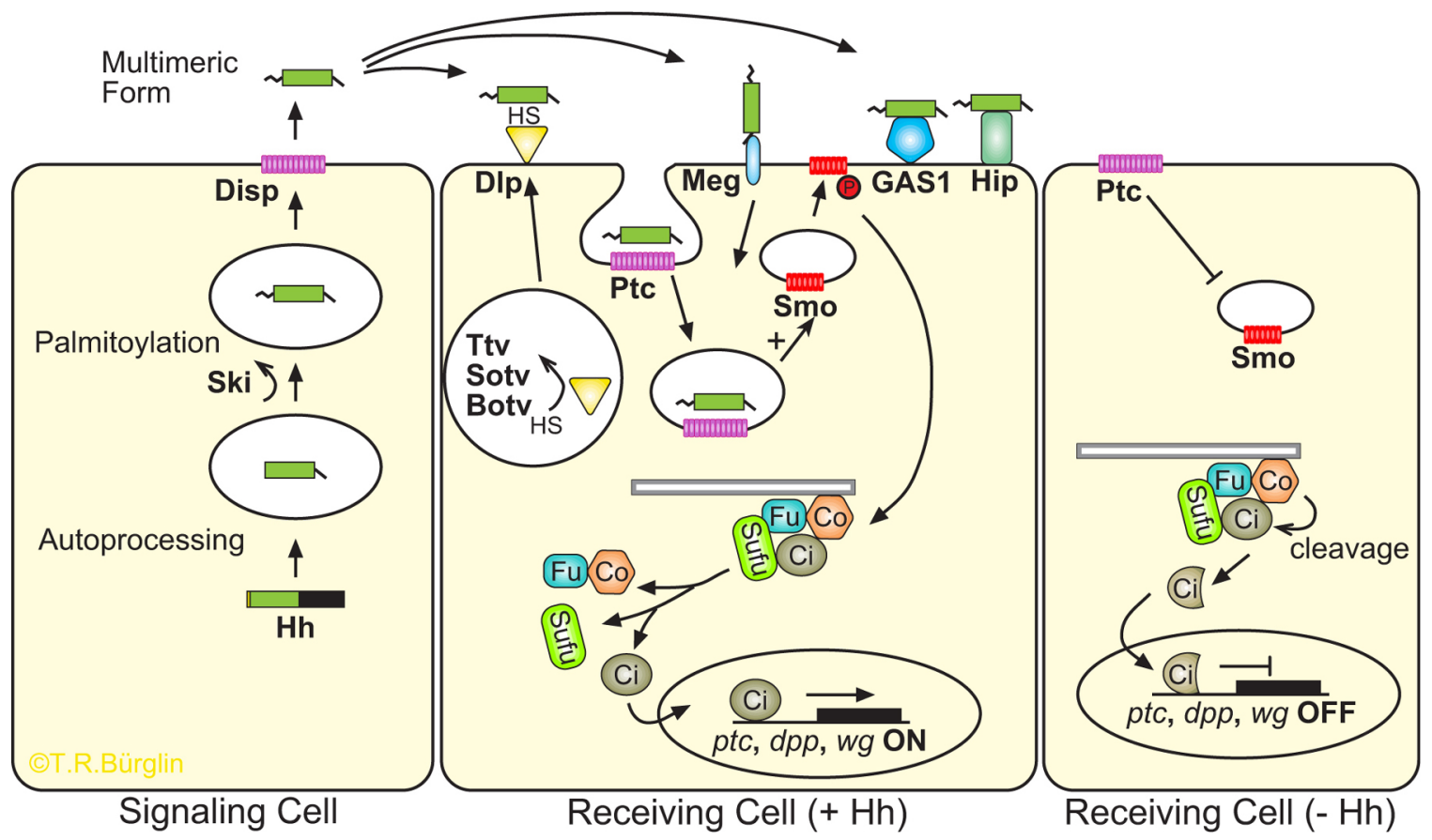

Figure 1. Hh encodes a secreted morphogen that activates a signal transduction pathway. Figure is based on studies from Drosophila and mammals and is adapted from Cohen (2003). A description of genes and abbreviations is provided (Table 1; for reviews, see Lee et al., 1992; Mohler and Vani, 1992; Tabata et al., 1992; Tashiro et al., 1993). Left panel: Hh is produced, autoprocessed and modified by Ski. N-Hh is released by Disp. Middle panel: The $\mathrm{N}-\mathrm{Hh}$ signal is propagated by the HSPG Dlp, which is modified by transferases such as Ttv, Sotv, Botv that add heparan sulphate (HS). Other molecules that can bind Hh signal are megalin, Hip and GAS1. N-Hh is an antagonistic ligand that represses the Patched (Ptc) receptor, before being internalised, and releases the latent activity of the Smoothened (Smo) seven-pass membrane protein, which is related to the Frizzled family of receptors (Alcedo et al., 1996; Chen and Struhl, 1998; Nusse, 2003; van den Heuvel and Ingham, 1996). In turn, Cos2 is released from microtubules and the Cos2/Fu/Su(fu)/Ci complex releases Ci, which can then enter the nucleus to activate transcription (Hooper, 2003; Jia et al., 2003; Lum et al., 2003; Ogden et al., 2003; Ruel et al., 2003). Several molecules involved in modifying the Cos $2 / / \mathrm{Fu} / \mathrm{Su}(\mathrm{fu}) / \mathrm{Ci}$ complex are not shown. Right panel: In the absence of N-Hh, Ptc inhibits Smo, the $\mathrm{Cos} 2 / \mathrm{Fu} / \mathrm{Su}(\mathrm{fu}) / \mathrm{Ci}$ complex remains associated with microtubules, $\mathrm{Ci}$ is phosphorylated and cleaved by $\mathrm{Cos} 2 . \mathrm{The} \mathrm{Ci}$ fragment now acts as a transcriptional repressor. Details of the pathway relevant for C. elegans are provided in the text. Grey bar represents microtubules. 


\section{Hh pathway components in C. elegans}

What role does the Hh/Ptc signalling pathway play in C. elegans development? To address this question, it is first reasonable to ask whether the major proteins of the pathway are encoded by the C. elegans genome, a task that is simplified by the availability of the complete genome sequence (Table 1; C. elegans Sequencing Consortium, 1998). Surprisingly, genes encoding recognisable Hh and Smo proteins are absent. In addition, homologs for Cos2, $\mathrm{Fu}$ and $\mathrm{Su}(\mathrm{fu})$, which transduce the $\mathrm{Hh}$ signal from $\mathrm{Smo}$ to $\mathrm{Ci}$, are absent. The $\mathrm{Ci}$ homolog itself, TRA-1, is not essential for embryonic pattern formation, but continues to act at the transcriptional level to control sexual cell fate decisions and male gonadal development (Hodgkin, 1983; Mathies et al., 2004), although there is no evidence that the nuclear localisation or activity of TRA-1 is controlled in the same manner as Ci (Kuwabara et al., 2000; Zarkower and Hodgkin, 1992). It has been speculated that the C. elegans sex determination pathway could be evolutionarily related to the $\mathrm{Hh} / \mathrm{Ptc}$ pathway because additional similarities have also been noted between the two pathways (Kuwabara et al., 1992).

Table 1. Components of the Hh signaling network. Note: the function of many of the C. elegans components is not clear and may be different from that in Drosophila and/or mammals. The abbreviations used for the hh pathway in Figure 1 are highlighted in yellow in this table. Slashes separate synonymous gene names, parentheses contain the abbreviations of the full gene names, comas separate paralogs.

\begin{tabular}{|c|c|c|c|}
\hline Role & C. elegans & Drosophila & Mammals \\
\hline \multicolumn{4}{|l|}{ Signal } \\
\hline $\begin{array}{c}\text { Hint and associated } \\
\text { domains }\end{array}$ & $\begin{array}{c}\text { qua-1, wrt-1 - wrt-10, grd-1 } \\
\text { - grd-17, grl-1 - grl-32, } \\
\text { hog-1 }\end{array}$ & hedgehog (hh) & $\begin{array}{l}\text { Sonic hedgehog (Shh), } \\
\text { Indian hedgehog (Ihh), } \\
\text { Desert hedgehog (Dhh) }\end{array}$ \\
\hline $\begin{array}{c}\text { Palmitoylation, membrane } \\
\text { bound O-acyl transferase, } \\
\text { MBOAT }\end{array}$ & Y57G11C.17, ZC101.3 & $\begin{array}{c}\text { rasp/skinny hedgehog } \\
(\text { ski }) / \text { sightless }\end{array}$ & Ski1/Skn/HHAT \\
\hline 12-Pass TM, SSD & che-14,ptd-2 & dispatched (disp) & DISP \\
\hline \multicolumn{4}{|l|}{ Signal modulators } \\
\hline $\begin{array}{l}\text { Hip module (homologs in } \\
\text { plants, bacteria) }\end{array}$ & - & - & $\begin{array}{l}\text { Hedgehog interacting } \\
\text { protein (Hipl/Hip) }\end{array}$ \\
\hline $\begin{array}{c}\text { GPI-anchored external } \\
\text { plasma membrane } \\
\text { glyco-protein (growth arrest } \\
\text { when overexpressed) }\end{array}$ & phg-1 & - & $\begin{array}{c}\text { Growth arrest specific } 1 \\
(\text { Gas } 1)\end{array}$ \\
\hline $\begin{array}{l}\text { Low density lipoprotein } \\
\text { receptor family }\end{array}$ & lrp-1 & & Megalin/gp330/LRP2 \\
\hline \multirow{3}{*}{$\begin{array}{c}\text { Glycosyltransferases } \\
\text { involved in Heparan sulfate } \\
\text { proteoglycan (HSPG) } \\
\text { synthesis, exostosin domain }\end{array}$} & $\begin{array}{l}\text { rib-1 (lacks C-term. } \\
\text { putative catalytic region } \\
\text { with DXD motif) }\end{array}$ & tout-velu (ttv) & EXT1 \\
\hline & $r i b-2$ & brother of tout-velu (botv) & EXTL3 \\
\hline & - & sister of tout-velu (sotv) & $E X T 2$ \\
\hline $\begin{array}{l}\text { Glypican: heparan sulfate } \\
\text { proteoglycan anchored to } \\
\text { the cell membrane by a } \\
\text { GPI-anchor }\end{array}$ & gpn-1 & dally-like (dlp/dly) & Glypican-6 \\
\hline \multicolumn{4}{|l|}{ Receptor } \\
\hline 12-Pass TM, SSD & $p t c-1, p t c-3$ & patched (ptc) & PTCH1, PTCH2 \\
\hline \multicolumn{4}{|l|}{ Signal transduction } \\
\hline 7TM serpentine receptors & - & smoothened (smo) & SMO \\
\hline
\end{tabular}




\begin{tabular}{|c|c|c|c|}
\hline Role & C. elegans & Drosophila & Mammals \\
\hline $\begin{array}{c}\text { Serine/threonine kinase } \\
\text { (homologs in plants) }\end{array}$ & - & fused (fu) & FU \\
\hline $\begin{array}{c}\text { Sufu domain (homologs in } \\
\text { bacteria) }\end{array}$ & - & Suppressor of fused (Su(fu)) & SUFU \\
\hline Kinesin-like & - & costal 2/costa (cos) & KIF27, KIF7 \\
\hline $\begin{array}{c}\text { Zinc finger transcription } \\
\text { factor }\end{array}$ & tra-1 & cubitus interruptus (ci) & Glil, Gli2, Gli3 \\
\hline
\end{tabular}

The C. elegans genome encodes an abundance of what will be collectively referred to as Hh-related (Hh-r) proteins, three Patched proteins and 24 Patched-related proteins (PTR). Four Frizzled receptor homologues (see Wnt signaling), as well as a series of molecules participating in the early stages of Hh signalling are also present. Given our current understanding of the $\mathrm{Hh} / \mathrm{Ptc}$ signal transduction pathway in other organisms, the presence of multiple $C$. elegans $\mathrm{Hh}-\mathrm{r}, \mathrm{PTC}$ and PTR proteins and the absence of $\mathrm{Hh}$, Smo and other components is unexpected and raises a number of questions about the evolution and the function of these genes in C.elegans.

\section{Hh-r genes, the structure}

The Hh proteins are composed of an $\mathrm{N}$-terminal signalling domain, $\mathrm{Hh}-\mathrm{N}$, and a $\mathrm{C}$-terminal autoprocessing domain, Hh-C. Hh-C has autoproteolytic activity, which cleaves $\mathrm{Hh}$ and subsequently attaches a cholesterol moiety to the Hh-N C-terminus (see scheme Figure 2A; Lee et al., 1994; Porter et al., 1996). Hh-C shares sequence similarity with the self-splicing inteins; hence, this region was named Hint (Dalgaard et al., 1997; Hall et al., 1997; Koonin, 1995). The 60 terminal residues of Hh-C are named the sterol recognition region (SRR) because they are required for adding cholesterol to Hh-N (Figure 2A; Beachy et al., 1997). Here, we refer to the combined Hint-SRR domain as the Hog domain.

Surprisingly, early database searches uncovered eight distinct $C$. elegans ORFs sharing similarity to Hh (Bürglin, 1996; Porter et al., 1996). Further investigation revealed that the sequence similarity shared by Hh and these ORFs was confined only to the Hog domain. Analyses of the N-terminal regions revealed several new motifs (Bürglin, 1996; Porter et al., 1996), which were also found in other ORFs that lacked Hog domains. In mimicry of the bipartite "Hedge-hog" structure, the three new gene families were named, warthog (wrt), groundhog (grd) and ground-like ( $\mathrm{grl}$ ) and the protein domains found at their respective N-terminal regions were called Wart, Ground and Ground-like (Aspöck et al., 1999; Bürglin, 1996). The final additions to the nomenclature are two previously unnamed single copy genes, qua-1 for quahog (T05C12.10/M110, Hao et al., 2006b), and an ORF consisting only of the Hog domain, hog-1 (W06B11.4). The structural organization of Hh and the Hh-r genes is shown in Figure 2B.

Over $60 \mathrm{Hh}-\mathrm{r}$ genes have been identified in C. elegans, most of which are expressed, although a few appear to be pseudogenes (e.g., grd-17; Figure 2B). Many of these genes share a one to one orthology to C. briggsae genes, although several have arisen through recent duplications within the $C$. elegans lineage. Preliminary searches of the ongoing Brugia malayi genome project reveal that representatives of the different $C$. elegans families are also present (Figure 2B). This clearly demonstrates that these Hh-r gene families were already present in the last common ancestor of $C$. elegans and B. malayi more than 300 million years ago.

The Wart, Ground, Ground-like and Qua domain each have a characteristic pattern of conserved cysteine residues (Figure 2C). The Ground and Ground-like domains differ in their arrangement of these conserved cysteine residues (Aspöck et al., 1999). In some instances, it has been possible to predict likely disulfide bond arrangements because of pairwise cysteine substitutions (Figure 2C; Aspöck et al., 1999). The Wart domain is about twice the size of the Ground and Ground-like domain; however, the C-terminal half of the Wart domain has a cysteine arrangement similar to that of the Ground domain and both share a short conserved motif similarly positioned between cysteine residues (FDøI, FEøV, respectively, Figure 2C; Aspöck et al., 1999). Thus, it is likely that the $\mathrm{C}$-terminal part of the Wart domain and the Ground domain was derived from the same ancestral domain. The short conserved FDøV "core" motif is also present in the Hh-N core, although its position relative to the cysteine residues is different (Figure 2C; Aspöck et al., 1999). 

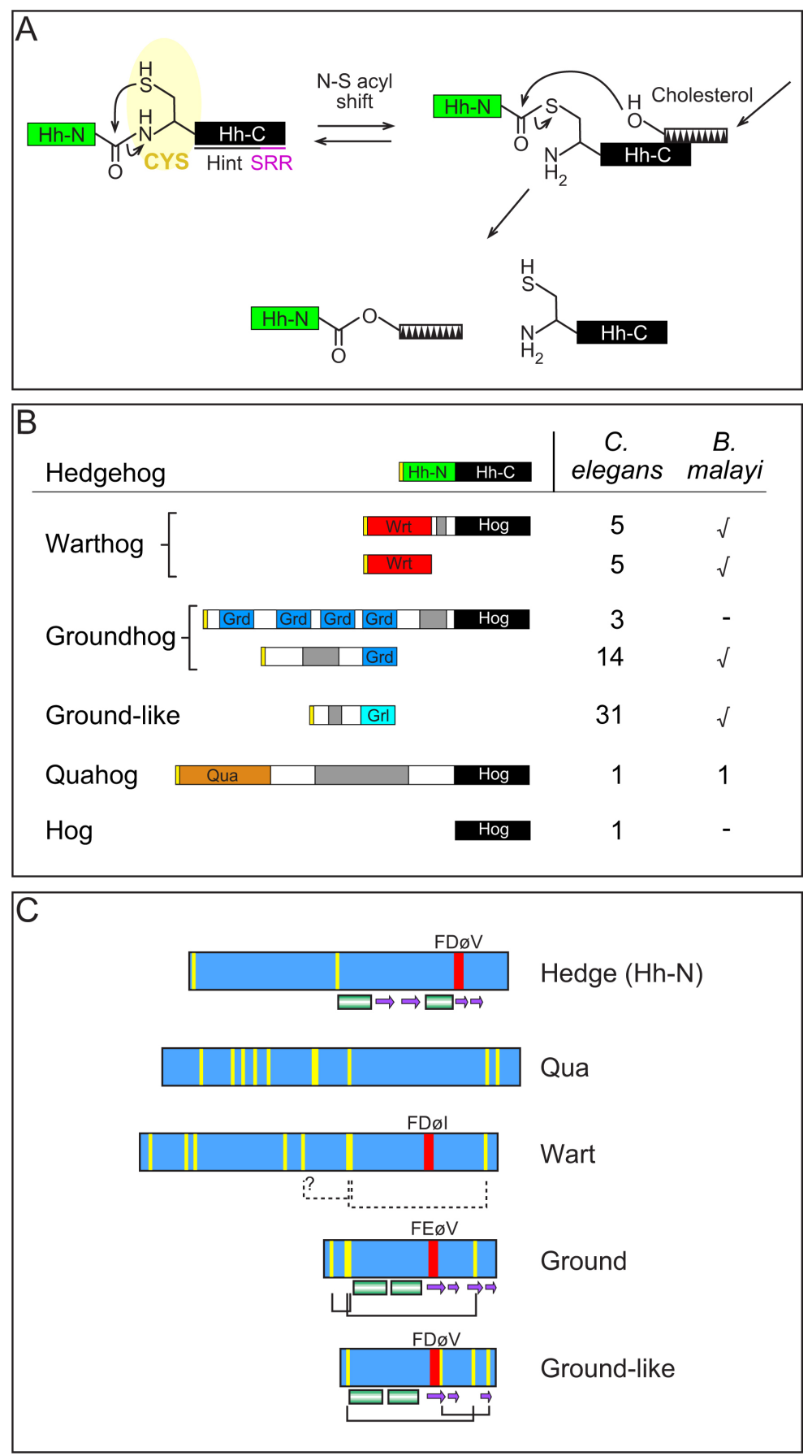

Figure 2. Structural features of the C. elegans Hh-r proteins . A) Intramolecular autoprocessing of Hh. The extent of the Hint domain and the sterol recognition region (SRR) is underlined beneath Hh-C; in C. elegans the SRR region within the Hog domain is called ARR, since it is unknown if it binds cholesterol (Beachy et al., 1997). The conserved first residue (cysteine) of the Hint domain is highlighted in yellow. The toothed box represents cholesterol. Acids and bases assisting in catalysis are not shown (adapted from Hall et al., 1997). B) Schematic representation of the conserved domains in Hh, WRT, GRD, GRL, QUA-1 and HOG-1 proteins. The yellow box at the N-terminus indicates the signal sequence for secretion and the black box represents the Hog domain. The green, red, blue, light blue and orange boxes indicate the Hh-N ("Hedge"), Wart, Ground, Ground-like, and Qua domains, respectively. The grey regions indicate regions with repetitive sequences of varying lengths. The first column on the right indicates the number of members of each family that are present in $C$. elegans. The second column shows a tick mark when members of these families have been detected in $B$. malayi, but the precise number has not yet been determined. C) Schematic representation of the Hh-N, Qua, Wart, Ground, and Ground-like domains. Yellow lines represent conserved cysteine residues. The red bar indicates a short conserved motif that often starts with a phenylalanine, followed by an acidic residue and two hydrophobic residues; $\varnothing$ stands for a small hydrophobic residue. Black solid lines beneath the domains mark predicted disulfide bonds, dashed lines are tentative predictions. The cylinders and arrows beneath $\mathrm{Hh}-\mathrm{N}$ indicate alpha helices and beta-sheets, respectively, taken from the solved 3D structure (Hall et al., 1995). Cylinders and arrows beneath the Ground and Ground-like domain were produced by secondary structure prediction of the multiple aligned sequences (Aspöck et al., 1999). 


\section{Modification and function of Hh-r genes}

All Hh-r genes in C. elegans, apart from hog-1, are predicted to encode secreted molecules. Porter et al. (1996) have shown that WRT-1, similar to Hh, undergoes autoprocessing; thus it is expected that other Hh-r proteins with a Hog domain will also have autocatalytic properties. However, it remains unclear whether the Hh-r proteins can bind cholesterol or other sterols, because the region corresponding to the SRR of Hh is slightly different in the Hh-r proteins (see Figure 1A in Aspöck et al., 1999).

An expression survey of wrt and grd genes revealed that they are expressed primarily in ectodermal tissues, particularly the hypodermis and seam cells, but can also be found in neurons, neuron-associated cells and gland cells (Aspöck et al., 1999). qua-1 is also expressed in the hypodermis (Hao et al., 2006b). An internal deletion in qua- 1 results in severe molting defects (Hao et al., 2006b). Functional studies of other wrt genes are in progress and preliminary results indicate that they also play important roles in development. For example, wrt-5 functions in the hypodermis during embryogenesis (Hao et al., 2006a). At present it is still unclear how Hh-r molecules function, and whether their activity could relate to the function of the PTC and PTR proteins.

In Drosophila, Hh is further modified by Skinny hedgehog (Ski), which palmitoylates the N-terminus of Hh-N, an essential process for Hh function (Chamoun et al., 2001). C. elegans has two co-orthologs, Y57G11C.17 and ZC101.3 (T.R. Bürglin, unpublished), but large-scale RNAi experiments have not uncovered any associated mutant phenotype. Modified Hh is secreted with the help of Dispatched; C. elegans has two Disp homologs, CHE-14 and PTD-2 (see below).

Secreted Hh forms a multimeric complex that moves through the extracellular matrix space. Heparan sulphate proteoglycans (HSPG) play an important role in Hh signal transduction. Three Drosophila genes, tout-velu (ttv), sister of tout-velu (sotv), and brother of tout-velu (botv) encode glycosyltransferases of the EXT family that are required for the biosynthesis of heparan sulfate and are essential for the movement of N-Hh (Han et al., 2004a; Takei et al., 2004). One target for these enzymes is Dally-like (Dlp), a glypican of the HSPG family that is also essential for Hh signalling (Desbordes and Sanson, 2003; Han et al., 2004b; Lum et al., 2003). C. elegans has two EXT family members, $r i b-1$ and rib-2 (for review, see Berninsone and Hirschberg, 2002). rib-1 is orthologous to ttv and EXT1, and rib-2 is ortholgous to botv and EXTL3; however, orthologs of other EXT members are absent (Figure 3). The EXT proteins have two domains: the exostosin domain and a 300 amino acid C-terminal region that is highly conserved between members and probably corresponds to the catalytic transferase domain, which is missing in $r i b-1$. Homozygous $r i b-2$ animals show delayed larval growth and reduced life span, while their progeny display more than 90\% early embryonic lethality (Morio et al., 2003). The C. elegans Dally-like gene ortholog, gpn-1, shows no phenotype in large-scale RNAi screens.

Two other molecules that bind and restrict the availability and range of $\mathrm{N}-\mathrm{Hh}$ are Hip and Growth arrest specific 1 (GAS1; for review, see Cohen, 2003). Hip is absent in both Drosophila and C. elegans. GAS1 is a GPI-linked protein identified by its ability to arrest cell cycle progression when overexpressed. The $C$. elegans ortholog phg-1/phas-1 is expressed in the pharynx and may play a similar role in mitotic cell cycle regulation (Agostoni et al., 2002). Megalin, a member of the family of low-density lipoprotein receptors, is a third protein that binds to N-Hh (see Cohen, 2003). The C. elegans homolog lrp-1 is expressed in the hypodermis; mutations in lrp-1 produce molting defects (Yochem et al., 1999). 


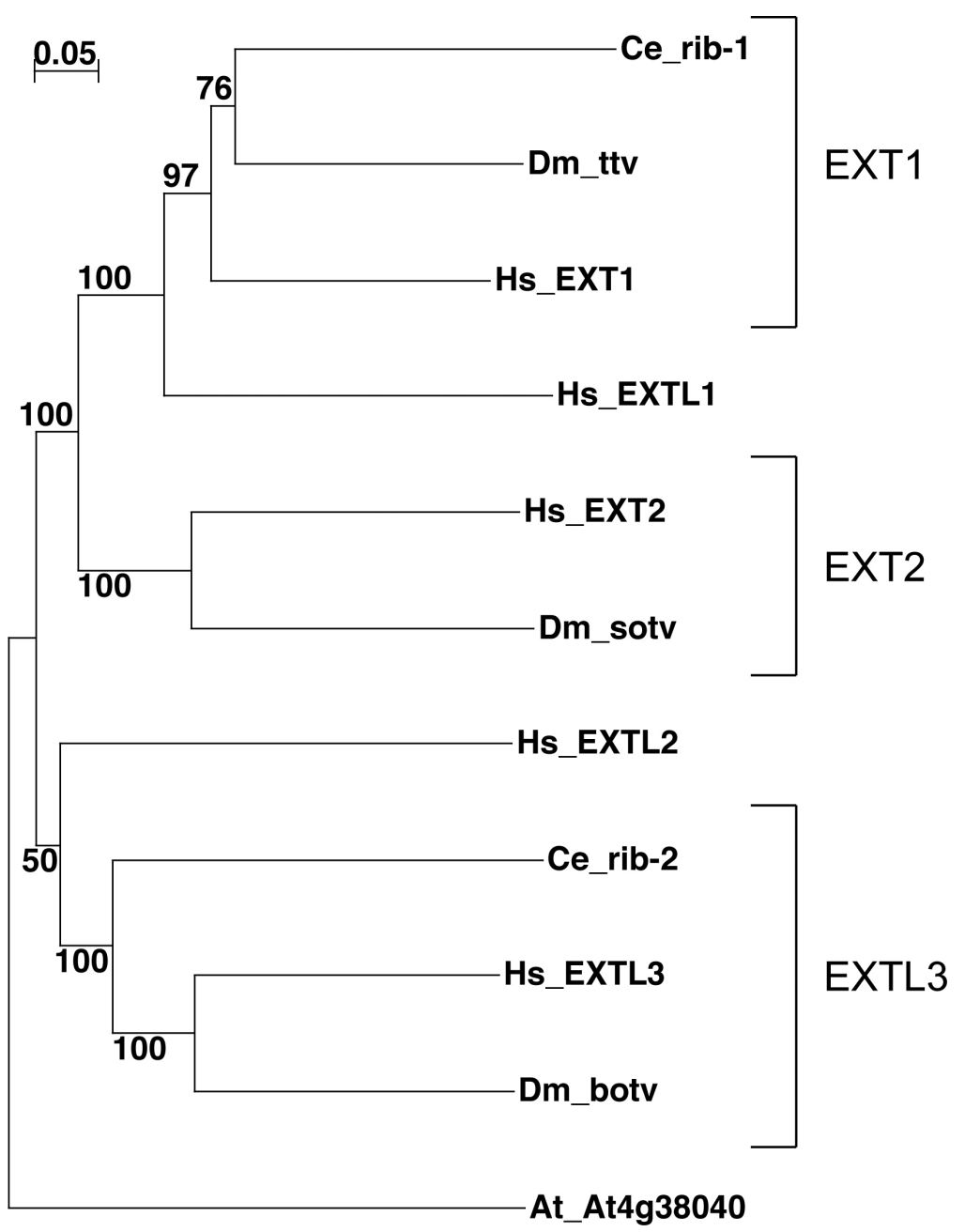

Figure 3. Phylogenetic tree of exostosin domain proteins from C. elegans $($ Ce $)$, Drosophila (Dm) and human (Hs). A multiple sequence alignment was produced using Clustal X (Thompson et al., 1997) and the built-in neighbor joining was used to generate a tree using the default values (not corrected for multiple substitutions, gaps not excluded). 1000 bootstrap trials were run, and trial values are shown in the figure as percentages. An Arabidopsis thaliana $(A t)$ exostosin domain containing protein was included as an outgroup. The three families conserved between flies and human are indicated.

\section{SSD Proteins: PTC and PTC-related proteins}

Three ptc genes, ptc-1,-2,-3 have been identified in C. elegans, although ptc-2 is absent in C. briggsae and is likely to be a pseudogene arising from a recent duplication of ptc-1. In addition, 24 genes have been identified that encode proteins sharing both sequence and topological similarities to PTC; these proteins have been named PTR (for Patched-related; Figure 4).

Drosophila, human and mouse are each predicted to encode a single PTR protein; thus the PTR family appears to have undergone an expansion in C. elegans (Kuwabara et al., 2000). Moreover, all 24 ptr genes are present in $C$. briggsae and share a one to one orthology with C. elegans. 


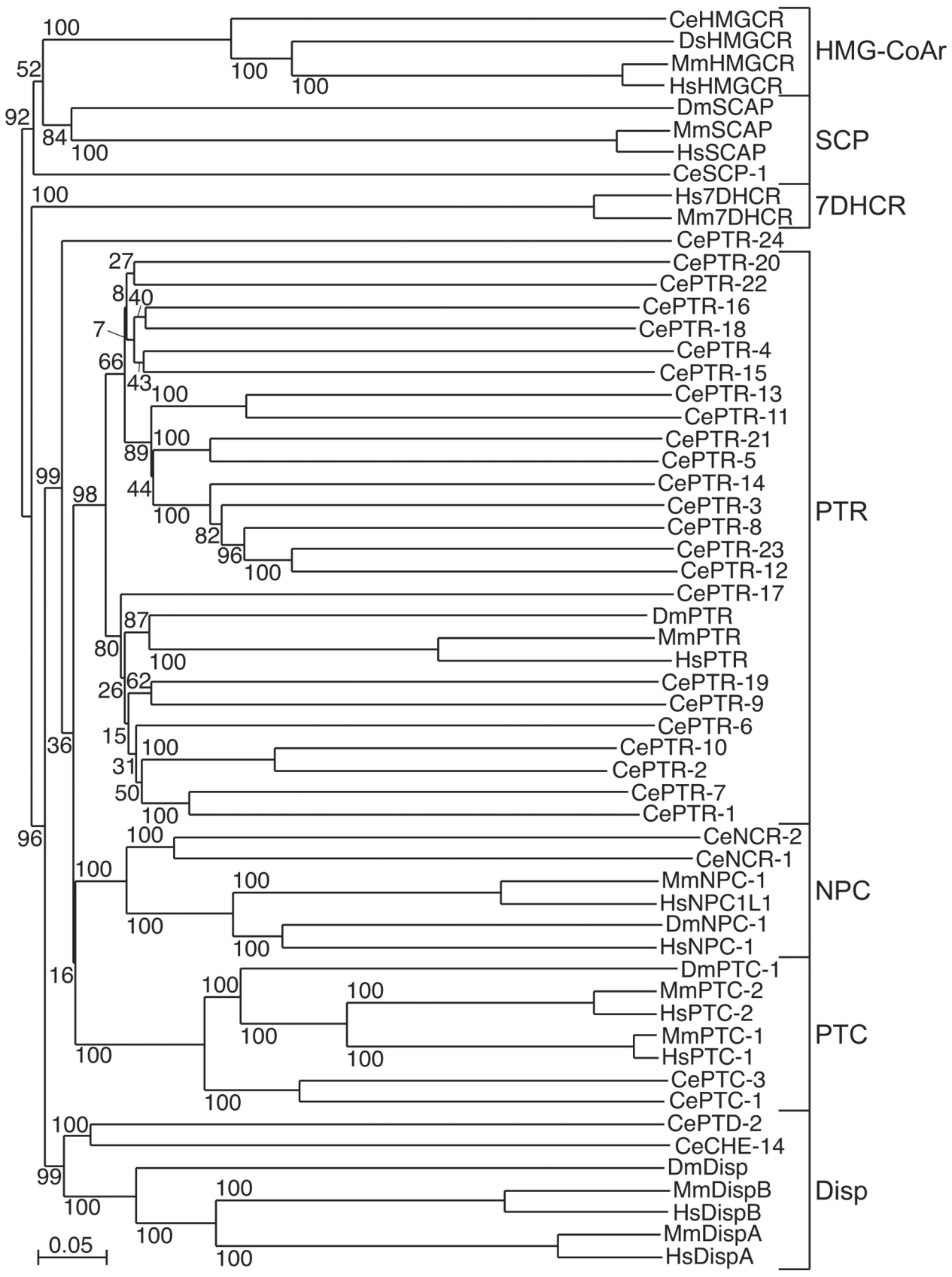

Figure 4. Phylogenetic tree of SSD proteins. A multiple sequence alignment of C. elegans (Ce), Drosophila (Dm) and human (Hs) SSD proteins was produced in Clustal X and the built-in neighbor joining was used to generate a tree using the default values (not corrected for multiple substitutions, gaps not excluded). 1000 bootstrap trials were run, and trial values are shown in the figure as percentages. Seven families of SSD proteins can be distinguished. Based on the bootstrap values, the PTC, NPC, Disp and PTR are more similar to each other. It is clear that the PTR family has expanded substantially in nematodes. PTR-24 does not fall within the clade of the PTR family, but we still believe it to be a divergent member of the PTR family. The PTR family itself seems to be composed of two subfamilies that each diverged and expanded to an equal extent. MSA data provided by O. Zugasti.

The C. elegans PTC and PTR proteins share characteristics common to all known Ptc proteins. The PTC and PTR proteins are predicted to have 12-membrane spanning domains with cytoplasmic $\mathrm{N}$ - and C-termini. The membrane domains can be further sub-divided into two cassettes of $1+5$, which are separated by a large intracellular loop. Each cassette contains a large extracellular loop predicted to facilitate Hh binding (Figure 5). Carried within the first set of TM domains is a sterol-sensing domain (SSD), which was first identified in SCAP, a protein involved in cholesterol homeostasis (Radhakrishnan et al., 2004). 


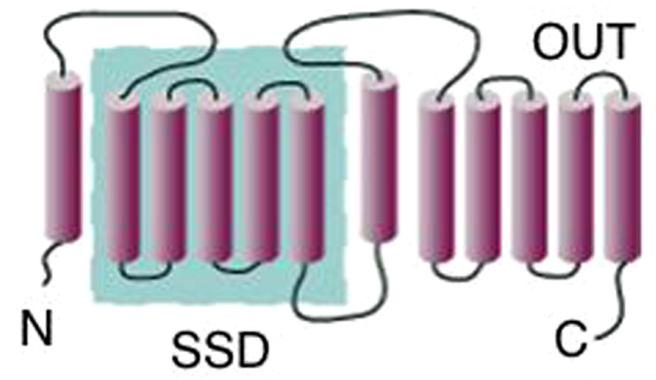

IN

Figure 5. Predicted topologies of PTC and PTR proteins. PTC and PTR proteins are predicted to encode proteins with 12-membrane spanning domains and share similarity with RND (Resistance, Nodulation, cell Division) bacterial multidrug transporter proteins (Hooper and Scott, 1989; Ingham et al., 1991; Kuwabara and Labouesse, 2002; Nakano et al., 1989). Blue inset highlights the five consecutive membrane-spanning domains that form the sterol-sensing domain (SSD).

\section{Role of PTC proteins in C. elegans}

ptc-1 is the first member of the ptc and ptr family to be analysed in detail (Kuwabara et al., 2000). Surprisingly, it was found that $p t c-1$ plays a crucial role in germline cytokinesis. Elimination of $p t c-1$ activity either by RNAi or mutation produces sterile adults that carry multinucleate oocytes and sperm. In other respects, development of a $p t c-1$ mutant is essentially wild type. In situ hybridization reveals that $p t c-1$ mRNA is maternally provided, and becomes enriched in $\mathrm{P} 4$, the germ line precursor. Immunocytochemistry performed using a polyclonal antibody capable of detecting PTC- 1 protein in wild type adult hermaphrodites reveals that PTC- 1 protein is enriched in the plasma membrane of mitotic germ cells undergoing proliferation, or in membranes undergoing rapid expansions, such as oocytes. PTC-1 protein is particularly enriched in vesicles at the apex of the membrane furrows separating individual germ nuclei.

An analysis of ptc- 1 mutants further revealed that the absence of cytokinesis generated membrane furrows leads to a loss of cell autonomous development in the germline syncytium, as evidenced by the presence of clusters of nuclei in M-phase (Kuwabara et al., 2000). The involvement of ptc-1 in cytokinesis has led to the hypothesis that PTC-1 could play a role in the transport of lipids, proteins or lipoproteins to the cleavage furrow. Importantly, a proteomic survey of proteins involved in mammalian cytokinesis reveals that Ptc-1 is a component of the mid-body (Skop et al., 2004). Hence, the involvement of PTC-1 in cytokinesis is likely to highlight an ancestral function for Ptc proteins, which is apparently independent of Smoothened.

A mutation has also been obtained for $p t c-3$. Preliminary phenotypic characterisation indicates that $p t c-3$ is an essential gene; mutants arrest in late embryogenesis (P.E.K., unpublished; Zugasti et al., 2005). A survey of the other ptr genes by RNAi reveals that many have roles in molting, but also affect growth and morphogenesis (Zugasti et al., 2005). It has also been demonstrated that C. elegans daf-6 encodes a Patched-related protein required for lumen formation (Perens and Shaham, 2005).

\section{Other SSD proteins provide clues to PTC and PTR function}

Another clue to the function of PTC-1 in worms is provided by the presence of an SSD and its connections to sterols and protein transport. The SSD of SCAP, the first identified SSD protein, is capable of binding directly to cholesterol (Radhakrishnan et al., 2004; Figure 4). Other SSD protein families that are closely related to PTC are the Dispatched family, which includes CHE-14 and PTD-2, and the Niemann-Pick Type C family, which includes the NCR-1, -2 proteins in C. elegans (Kuwabara and Labouesse, 2002). che-14 was first identified in a screen for animals that were defective in dye-filling (Perkins et al., 1986). Subsequent work demonstrated that che-14 promotes apical sorting in epithelial cells. che-14 mutants accumulate vesicles that are trapped near the apical surface, indicative of problems in protein secretion (Michaux et al., 2000). The phenotype of che-14 mutants reveals that the problem is more likely to be associated with defects in exocytosis, rather than in endocytosis. The presence of an SSD in CHE-14 led the authors to speculate that CHE-14 might be involved in the secretion of proteins containing sterol or GPI adducts. Similarly, Disp promotes the exocytosis of Hh away from the cells from which it was synthesised (Burke et al., 1999). A protein transported by CHE-14 might be WRT-6, which is expressed in sensory socket cells (Aspöck et al., 1999). 
Niemann-Pick disease type C1 (NPC1) is a neurodegenerative disease associated with defects in cholesterol storage/transport. In $C$. elegans, $n c r-1 ; n c r-2$ double mutants are hypersensitive to cholesterol deprivation and inappropriately form dauers under favourable food conditions, a dauer-constitutive (Daf-c) phenotype (Sym et al., 2000). Recent studies further show that $n c r-1$ is hypersensitive to progesterone, a known inhibitor of cholesterol trafficking (Li et al., 2004). Hence, in C. elegans there is a strong link between SSD proteins and the transport of sterols, sterol modified proteins or in the case of dauer formation, a sterol modified hormone.

C. elegans are cholesterol auxotrophs, requiring exogenous cholesterol for growth and survival (Chitwood, 1999; Hieb and Rothstein, 1968). Sterols are involved in at least two processes in the worm: the decision to enter diapause, which is mediated by a sterol-derived hormone, and molting, which is impaired by sterol depletion (Matyash et al., 2004). Animals depleted for cholesterol arrest in the second generation during early larval stages (Matyash et al., 2001; Merris et al., 2003). These L2 arrested larvae became dauer-like and fail to complete molting (Matyash et al., 2004).

Because worms require so relatively little cholesterol, it has been postulated that cholesterol is unlikely to be an essential architectural component of membranes and could play a more important role in signalling (Matyash et al., 2001). Mutations in lrp-1, qua-1 and RNAi inhibition of most ptr genes, and post-embryonic RNAi of ptc gene activity all lead to defects in molting (Yochem et al., 1999; Zugasti et al., 2005; Hao et al., 2006b). Thus, taken together, it is speculated that the PTC and PTR proteins could promote the transport of sterols or sterol-modified proteins, possibly including sterol-modified Hh-r proteins.

\section{Evolution}

C. elegans lacks the typical Hh signal transduction cascade found in flies and vertebrates that is composed of $\mathrm{Smo}, \mathrm{Fu}, \mathrm{Su}(\mathrm{fu})$, and $\mathrm{Cos} 2$. Since homologs of $\mathrm{Fu}$ and $\mathrm{Su}(\mathrm{fu})$ are found in plants and bacteria (T.R.B., unpublished), it follows that this part of the pathway was lost in nematodes. Concomitantly, we have observed a large increase in the number of $C$. elegans Hh-r and ptr genes. Several lines of evidence point towards the Hh-r expansion being nematode specific: 1) Homologs of the Hh-r genes have so far not been identified outside of the nematodes. 2) Conversely, $\mathrm{Hh}$ is present in both arthropods and vertebrates and only missing in nematodes. 3) Phylogenetic analyses of the Hog domain and motif similarity shared by the Wrt and Grd domains indicate that the wrt, grd and $\mathrm{grl}$ gene families are derived from a single ancestral gene (Aspöck et al., 1999); qua-1 may also be derived from the same ancestral gene, as its Hog domain is also more similar to those of the Wrt and Grd proteins than to that of Hh. 4) C. briggsae has fewer Hh-r genes than C. elegans, and B. malayi even fewer, which underscores the capacity of these genes to expand. The expansion of the ptr genes is likewise nematode specific, since Drosophila and humans have only a single copy.

There is a running debate whether or not nematodes should be classified as ecdysozoa. If nematodes are indeed ecdysozoa, or group at least within the protostome branch of ecdysozoa and lophotrochozoa (see chapter on evolution), then the loss, expansion and diversification of various Hh pathway components, which have been described here, are most parsimoniously explained by postulating that a C. elegans Hh signalling pathway did exist, but was disrupted at some unknown time in early nematode evolution. It also implies that the Hh-r genes were derived from Hh. On the other hand, if nematodes are an outgroup to coelomata, then one of several alternatives is that a complete Hh signalling pathway was never acquired.

\section{Summary}

It is clear that the Hh and Ptc homologs in C. elegans have undergone considerable evolutionary divergence when compared to their Drosophila and vertebrate counterparts. Studies in C. elegans are providing insights into the roles and mechanisms underlying the control of the Hh-r, PTC and PTC-related proteins and have established that these proteins can function independently of Smo. The involvement of Hh-r and ptr genes in molting suggests that at least some of the Hh-r and ptr genes can participate in similar pathways. Thus, it will be of particular interest to determine whether the Hh-r proteins and the PTC and PTR proteins have maintained a ligand/receptor relationship that could potentially mediate cell-cell signalling. 


\section{Acknowledgements}

TRB would like to thank the Swedish Foundation for Strategic Research (SSF) for support. PEK thanks the MRC (UK) for funding. The authors would like to thank David Fitch for constructive discussions related to nematode phylogeny.

\section{References}

Agostoni, E., Gobessi, S., Petrini, E., Monte, M., and Schneider, C. (2002). Cloning and characterization of the $C$. elegans gas1 homolog: phas-1. Biochim. Biophys. Acta 1574, 1-9. Abstract

Alcedo, J., Ayzenzon, M., Von Ohlen, T., Noll, M., and Hooper, J.E. (1996). The Drosophila smoothened gene encodes a seven-pass membrane protein, a putative receptor for the hedgehog signal. Cell 86, 221-232. Abstract Article

Aspöck, G., Kagoshima, H., Niklaus, G., and Bürglin, T.R. (1999). Caenorhabditis elegans has scores of hedgehog-related genes: sequence and expression analysis. Genome Res. 9, 909-923. Abstract Article

Bale, A.E. (2002). Hedgehog signaling and human disease. Annu. Rev. Genomics Hum. Genet. 3, 47-65. Abstract Article

Beachy, P.A., Cooper, M.K., Young, K.E., von Kessler, D.P., Park, W.J., Hall, T.M., Leahy, D.J., and Porter, J.A. (1997). Multiple roles of cholesterol in hedgehog protein biogenesis and signaling. Cold Spring Harb. Symp. Quant. Biol. 62, 191-204. Abstract

Beachy, P.A., Karhadkar, S.S., and Berman, D.M. (2004). Tissue repair and stem cell renewal in carcinogenesis. Nature 432, 324-331. Abstract Article

Berninsone, P.M., and Hirschberg, C.B. (2002). The nematode Caenorhabditis elegans as a model to study the roles of proteoglycans. Glycoconj J. 19, 325-330. Abstract Article

Bürglin, T.R. (1996). Warthog and Groundhog, novel families related to Hedgehog. Curr. Biol. 6, 1047-1050. Abstract

Burke, R., Nellen, D., Bellotto, M., Hafen, E., Senti, K.A., Dickson, B.J., and Basler, K. (1999). Dispatched, a novel sterol-sensing domain protein dedicated to the release of cholesterol-modified hedgehog from signaling cells. Cell 99, 803-815. Abstract Article

C. elegans Sequencing Consortium (1998). Genome sequence of the nematode C. elegans: a platform for investigating biology. Science 282, 2012-2018. Abstract Article

Chamoun, Z., Mann, R.K., Nellen, D., von Kessler, D.P., Bellotto, M., Beachy, P.A., and Basler, K. (2001). Skinny hedgehog, an acyltransferase required for palmitoylation and activity of the hedgehog signal. Science 293, 2080-2084. Abstract Article

Chen, Y., and Struhl, G. (1998). In vivo evidence that Patched and Smoothened constitute distinct binding and transducing components of a Hedgehog receptor complex. Development 125, 4943-4948. Abstract

Chitwood, D.J. (1999). Biochemistry and function of nematode steroids. Crit. Rev. Biochem. Mol. Biol. 34, 273-284. Abstract Article

Cohen, M.M., Jr. (2003). The hedgehog signaling network. Am. J. Med. Genet. A 123, 5-28. Abstract Article

Dalgaard, J.Z., Moser, M.J., Hughey, R., and Mian, I.S. (1997). Statistical modeling, phylogenetic analysis and structure prediction of a protein splicing domain common to inteins and hedgehog proteins. J. Comput. Biol. 4, 193-214. Abstract 
Desbordes, S.C., and Sanson, B. (2003). The glypican Dally-like is required for Hedgehog signalling in the embryonic epidermis of Drosophila. Development 130, 6245-6255. Abstract Article

Hall, T.M., Porter, J.A., Beachy, P.A., and Leahy, D.J. (1995). A potential catalytic site revealed by the $1.7-\AA$ crystal structure of the amino-terminal signalling domain of Sonic hedgehog. Nature 378, 212-216. Abstract Article

Hall, T.M., Porter, J.A., Young, K.E., Koonin, E.V., Beachy, P.A., and Leahy, D.J. (1997). Crystal structure of a Hedgehog autoprocessing domain: homology between Hedgehog and self-splicing proteins. Cell 91, 85-97. Abstract Article

Han, C., Belenkaya, T.Y., Khodoun, M., Tauchi, M., and Lin, X. (2004a). Distinct and collaborative roles of Drosophila EXT family proteins in morphogen signalling and gradient formation. Development 131, 1563-1575. Abstract Article

Han, C., Belenkaya, T.Y., Wang, B., and Lin, X. (2004b). Drosophila glypicans control the cell-to-cell movement of Hedgehog by a dynamin-independent process. Development 131, 601-611. Abstract Article

Hao, L., Aspöck, G., and Bürglin, T.R. (2006a). The hedgehog-related gene wrt-5 is essential for hypodermal development in Caenorhabditis elegans. Dev. Biol. in press.

Hao, L., Mukherjee, K., Liegeois, S., Baillie, D., Labouesse, M., and Bürglin, T.R. (2006b). The hedgehog-related gene qua-1 is required for molting in Caenorhabditis elegans. Dev. Dynamics, in press.

Hieb, W.F., and Rothstein, M. (1968). Sterol requirement for reproduction of a free-living nematode. Science 160, 778-780. Abstract

Hodgkin, J. (1983). Two types of sex determination in a nematode. Nature 304, 267-268. Abstract Article

Hooper, J.E. (2003). Smoothened translates Hedgehog levels into distinct responses. Development 130, 3951-3963. Abstract Article

Hooper, J.E., and Scott, M.P. (1989). The Drosophila patched gene encodes a putative membrane protein required for segmental patterning. Cell 59, 751-765. Abstract Article

Ingham, P.W., and McMahon, A.P. (2001). Hedgehog signaling in animal development: paradigms and principles. Genes Dev. 15, 3059-3087. Abstract Article

Ingham, P.W., Taylor, A.M., and Nakano, Y. (1991). Role of the Drosophila patched gene in positional signalling. Nature 353, 184-187. Abstract Article

Jia, J., Tong, C., and Jiang, J. (2003). Smoothened transduces Hedgehog signal by physically interacting with Costal2/Fused complex through its C-terminal tail. Genes Dev. 17, 2709-2720. Abstract Article

Koonin, E.V. (1995). A protein splice-junction motif in hedgehog family proteins. Trends Biochem. Sci. 20, 141-142. Abstract Article

Kuwabara, P.E., and Labouesse, M. (2002). The sterol-sensing domain: multiple families, a unique role? Trends Genet. 18, 193-201. Abstract Article

Kuwabara, P.E., Lee, M.H., Schedl, T., and Jefferis, G.S. (2000). A C. elegans patched gene, ptc-1, functions in germ-line cytokinesis. Genes Dev. 14, 1933-1944. Abstract

Kuwabara, P.E., Okkema, P.G., and Kimble, J. (1992). tra-2 encodes a membrane protein and may mediate cell communication in the Caenorhabditis elegans sex determination pathway. Mol. Biol. Cell 3, 461-473. Abstract

Lee, J.J., Ekker, S.C., von Kessler, D.P., Porter, J.A., Sun, B.I., and Beachy, P.A. (1994). Autoproteolysis in hedgehog protein biogenesis. Science 266, 1528-1537. Abstract 
Lee, J.J., von Kessler, D.P., Parks, S., and Beachy, P.A. (1992). Secretion and localized transcription suggest a role in positional signaling for products of the segmentation gene hedgehog. Cell 71,33-50. Abstract Article

Li, J., Brown, G., Ailion, M., Lee, S., and Thomas, J.H. (2004). NCR-1 and NCR-2, the C. elegans homologs of the human Niemann-Pick type C1 disease protein, function upstream of DAF-9 in the dauer formation pathways. Development 131, 5741-5752. Abstract Article

Lum, L., and Beachy, P.A. (2004). The Hedgehog response network: sensors, switches, and routers. Science 304, 1755-1759. Abstract Article

Lum, L., Yao, S., Mozer, B., Rovescalli, A., von Kessler, D., Nirenberg, M., and Beachy, P.A. (2003). Identification of Hedgehog pathway components by RNAi in Drosophila cultured cells. Science 299, 2039-2045. Abstract Article

Lum, L., Zhang, C., Oh, S., Mann, R.K., von Kessler, D.P., Taipale, J., Weis-Garcia, F., Gong, R., Wang, B., and Beachy, P.A. (2003). Hedgehog signal transduction via Smoothened association with a cytoplasmic complex scaffolded by the atypical kinesin, Costal-2. Mol Cell 12, 1261-1274. Abstract Article

Mathies, L.D., Schvarzstein, M., Morphy, K.M., Blelloch, R., Spence, A.M., and Kimble, J. (2004). TRA-1/GLI controls development of somatic gonadal precursors in C. elegans. Development 131, 4333-4343. Abstract Article

Matyash, V., Entchev, E.V., Mende, F., Wilsch-Bräuninger, M., Thiele, C., Schmidt, A.W., Knölker, H.J., Ward, S., and Kurzchalia, T.V. (2004). Sterol-derived hormone(s) controls entry into diapause in Caenorhabditis elegans by consecutive activation of DAF-12 and DAF-16. PLoS Biol. 2, e280. Abstract Article

Matyash, V., Geier, C., Henske, A., Mukherjee, S., Hirsh, D., Thiele, C., Grant, B., Maxfield, F.R., and Kurzchalia, T.V. (2001). Distribution and transport of cholesterol in Caenorhabditis elegans. Mol. Biol. Cell 12, 1725-1736. Abstract

Merris, M., Wadsworth, W.G., Khamrai, U., Bittman, R., Chitwood, D.J., and Lenard, J. (2003). Sterol effects and sites of sterol accumulation in Caenorhabditis elegans: developmental requirement for 4alpha-methyl sterols. J. Lipid Res. 44, 172-181. Abstract Article

Michaux, G., Gansmuller, A., Hindelang, C., and Labouesse, M. (2000). CHE-14, a protein with a sterol-sensing domain, is required for apical sorting in C. elegans ectodermal epithelial cells. Curr. Biol. 10, 1098-1107. Abstract Article

Mohler, J., and Vani, K. (1992). Molecular organization and embryonic expression of the hedgehog gene involved in cell-cell communication in segmental patterning of Drosophila. Development 115, 957-971. Abstract

Morio, H., Honda, Y., Toyoda, H., Nakajima, M., Kurosawa, H., and Shirasawa, T. (2003). EXT gene family member rib-2 is essential for embryonic development and heparan sulfate biosynthesis in Caenorhabditis elegans. Biochem. Biophys. Res. Commun. 301, 317-323. Abstract Article

Nakano, Y., Guerrero, I., Hidalgo, A., Taylor, A., Whittle, J.R., and Ingham, P.W. (1989). A protein with several possible membrane-spanning domains encoded by the Drosophila segment polarity gene patched. Nature 341, 508-513. Abstract Article

Nusse, R. (2003). Wnts and Hedgehogs: lipid-modified proteins and similarities in signaling mechanisms at the cell surface. 130,5297-5305. Abstract

Nüsslein-Volhard, C., and Wieschaus, E. (1980). Mutations affecting segment number and polarity in Drosophila. Nature 287, 795-801. Abstract Article

Ogden, S.K., Ascano, M., Jr., Stegman, M.A., Suber, L.M., Hooper, J.E., and Robbins, D.J. (2003). Identification of a functional interaction between the transmembrane protein Smoothened and the kinesin-related protein Costal2. Curr. Biol. 13, 1998-2003. Abstract Article

Pasca di Magliano, M., and Hebrok, M. (2003). Hedgehog signalling in cancer formation and maintenance. Nat. Rev. Cancer 3, 903-911. Abstract Article 
Perens, E.A. and Shaham, S. (2005). C. elegans daf-6 encodes a Patched-related protein required for lumen formation. Dev. Cell 8, 893-906. Abstract Article

Perkins, L.A., Hedgecock, E.M., Thomson, J.N., and Culotti, J.G. (1986). Mutant sensory cilia in the nematode $C$. elegans. Dev. Biol. 117, 456-487. Abstract Article

Porter, J.A., Ekker, S.C., Park, W.J., von Kessler, D.P., Young, K.E., Chen, C.H., Ma, Y., Woods, A.S., Cotter, R.J., Koonin, E.V., and Beachy, P.A. (1996). Hedgehog patterning activity: role of a lipophilic modification mediated by the carboxy-terminal autoprocessing domain. Cell 86, 21-34. Abstract Article

Porter, J.A., Young, K.E., and Beachy, P.A. (1996). Cholesterol modification of hedgehog signaling proteins in animal development. Science 274, 255-259. Article

Radhakrishnan, A., Sun, L.P., Kwon, H.J., Brown, M. SS., and Goldstein, J.L. (2004). Direct binding of cholesterol to the purified membrane region of SCAP: mechanism for a sterol-sensing domain. Mol. Cell 15, 25968. Abstract Article

Ruel, L., Rodriguez, R., Gallet, A., Lavenant, L., and Therond, P.P. (2003). Stability and association of Smoothened, Costal2 and Fused with Cubitus interruptus are regulated by Hedgehog. Nat. Cell Biol. 5, 907-913. Abstract Article

Skop, A.R., Liu, H., Yates, J., 3rd, Meyer, B.J., and Heald, R. (2004). Dissection of the mammalian midbody proteome reveals conserved cytokinesis mechanisms. Science 305, 61-66. Abstract Article

Sym, M., Basson, M., and Johnson, C. (2000). A model for Niemann-Pick type C disease in the nematode Caenorhabditis elegans. Curr. Biol. 10, 527-530. Abstract Article

Tabata, T., Eaton, S., and Kornberg, T.B. (1992). The Drosophila hedgehog gene is expressed specifically in posterior compartment cells and is a target of engrailed regulation. Genes Dev. 6, 2635-2645. Abstract

Taipale, J., and Beachy, P.A. (2001). The Hedgehog andd Wnt signalling pathways in cancer. Nature 411, 349-354. Abstract Article

Takei, Y., Ozawa, Y., Sato, M., Watanabe, A., and Tabatta, T. (2004). Three Drosophila EXT genes shape morphogen gradients through synthesis of heparan sulfate proteoglycans. Development 131, 73-82. Abstract Article

Tashiro, S., Michiue, T., Higashijima, S., Zenno, S., Ishimaru, S., Takahashi, F., Orihara, M., Kojima, T., and Saigo, K. (1993). Structure and expression of hedgehog, a Drosophila segment-polarity gene required for cell-cell communication. Gene 124, 183-189. Abstract Article

Thompson, J.D., Gibson, T.J., Plewniak, F., Jeanmougin, F., and Higgins, D.G. (1997). The CLUSTAL_X windows interface: flexible strategies for multiple sequence alignment aided by quality analysis tools. Nucleic Acids Res. 25, 4876-4882. Abstract Article

van den Heuvel, M., and Ingham, P.W. (1996). Smoothened encodes a receptor-like serpentine protein required for hedgehog signalling. Nature 382, 547-551. Abstract Article

Yochem, J., Tuck, S., Greenwald, I., and Han, M. (1999). A gp330/megalin-related protein is required in the major epidermis of Caenorhabditis elegans for completion of molting. Development 126, 597-606. Abstract

Zarkower, D., and Hodgkin, J. (1992). Molecular analysis of the C. elegans sex-determining gene tra-1: a gene encoding two zinc finger proteins. Cell 70, 237-249. Abstract Article

Zugasti, O., Rajan, J., and Kuwabara, P.E. (2005). The function and expansion of the Patched- and Hedgehog-related homologs in C. elegans. Genome Res. 15, 1402-1410. Abstract Article

All WormBook content, except where otherwise noted, is licensed under a Creative

SOMIEREIGHISRESERVED Commons Attribution License. 